\title{
Comparison of Health Care System of China and Yemen
}

\author{
Aljaifi Saddam Hussein Mohammed \\ Master Degree Candidate, School of Health Sciences, Wuhan University, China, 430071
}

\begin{abstract}
Health is one of the basic requirements of the nation and the developing nations required introduced reforms into the health care system to ensure better health care facilities. The presence status of health care centers in the prime parameter to judge the human health index and indirectly define the country's prosperity. China is the leading developing nation with the highest growth rate of the human index, and the health care reforms are significant to boost up the health care facilities of its citizens. Although Yemen's government respectively has been providing a free healthcare services to its people, still it lacks basic public health care infrastructure which is far behind the average of the world health quality standers. we consolidated the comparison of public health between China and Yemen, recommended some health care reforms for Yemen by keeping the Chinese health system as a model, especially after Covid19 pandemic occurrence the world recognized the power and the potential of the china healthcare system in managing the outbreak. The quantitative and qualitative data analysis revealed that Chinese health care system is semi saturated with the presence of modern medical treatment, equipment and workers professionalism in its major cities. The Yemenis public health care system is much disturbed due to country political situation, lack of funding, and the basic medical facilities are even poor at major cities. Thus, people expressed high sort of dissatisfaction from the current health care system. Combined with the effectiveness of the strategies china's adopt, this study would shed light on the needs and encourage Yemeni to model china's health care strategy to create better public health reforms.
\end{abstract}

Keywords: healthcare facilities, patient security, economic influence, China, Yemen

DOI: $10.7176 / \mathrm{JHMN} / 73-03$

Publication date: April $30^{\text {th }} 2020$

\section{Introduction}

Although china and Yemen don't have much in common, both countries are in the process of undergoing healthcare reform in deferent level of the development. Over the precedent 30 years, both nations have considering how to achieve in health to improve life anticipation, condensed newborn mortality, and health care for disease control(Alssamei et al., 2017). In 2009, Chinese government represented by the communist party had proclaimed and established the health care reform, at the same year 2009, with considering a significant gap between the both countries different economy levels, Yemen has launched an officially inaugurated health care reforms trying to keep following a practical steps that the developing countries did to enhance and reinvestment in several different aspects of the healthcare quality. After a brief comparing the two healthcare systems reforms, now we referred to a cross-cutting themes; national development objectives is having a real impact on healthcare systems, the government structure, and economic climate. China's healthcare system is on the edge of most important improvement, enthused by a huge number of forces that are motivating this alteration. Since 2002, in an attempt to recover the superiority of patient care and patient confidentiality, the government of china has had the first and foremost allotted its healthcare financial support to former students of medical schools along with public hospitals in main cities and impalement the yearly health workers training. After the 2003, government assurance to develop health services as well as patient contentment was additional underlined. SARS occurrence, at the time of 2005 the "Year of Hospital Management Reform" was confirmed, with the important subject of "the highest priorities is patient care, amelioration the service level." (Baidoun, Salem, \& Omran, 2018; Balan et al., 2018) These measures make understandable of the government's dedication to rising hospital administration and association in a patient-centered method and to augment patient contentment. During the current pandemic outbreak COVID19 which sustained spread everywhere around the world china healthcare system been able to deal with it, shows the great potential to contain the pandemic. At this moment Chinese hospitals are working in an original, multifaceted and tentative environment to increase the critical care medicine ICU beds which was and still under development.(Bin Du* ICU management \& practice). The current alteration for a competitive healthcare marketplace is considering hospital contributors. Encounter these circumstances, contributors ought to find the most effectively to persuade requirements and wishes for patients security. Therefore, Contributors representatives both were immediately looking for an obvious consideration of the superiority, contentment and faithfulness intention association in the Chinese healthcare marketplace (Cunningham, Ingram, Brinckmann, \& Nesbitt, 2018). While in Yemen, patient security has been a severe worldwide public health matter. Approximation depicts that in emerging economies as several as one in 10 patients is debilitated while getting hospital care. There were 7 patients in an urbanized per 100 admitted versus 10 patient in an emergent countries getting openly obtain health carerelated infection. Most of people are deprived of sufficient access to secure and suitable medical devices within 
their health systems. Above half of low and lower middle earning nations do not possess a national health technology strategy which could make sure the efficient utilization of resources through correct scheduling, appraisal, attainment and administration of medical devices (Mozooni, Preen, \& Pennell, 2018). The Yemenis government have been giving a broad array to improve healthcare services to its citizens over a long period of time. Regardless the important plan, political disturbance has emerged serious problems in health care system over the previous years, created the rigorous immaturity in health care system. Concomitantly, the total per individual expense was minimal; in contrast with other Middle Eastern countries that is the lowest in accordance with United Nations information and the WHO. In accordance with the World Bank, "in the time between 1995 and 2000 Yemeni doctors has been increased, the number raised by an average of 7 percent, but unfortunately in 2004 The number of doctors has been decreased to a three doctor per 10,000 people. In 2003 the availability of Yemen hospital beds was barely 0.6 per 10,000 inhabitants, all those evidences indicated to the low health care system in Yemen, so we can imagine the scary serviced are in rural areas. Only one quarter of the total percent of rural areas were getting a serviced from healthcare foundations, as compared with 80 percent of urban areas" (Al-Murtadha \& Feryok, 2017). Quality of healthcare system has been the huge issue of nowadays that is almost the issue of every developed country. However, regardless of these achievements, the health condition of inhabitants of china still stays away from that, the country health achievements has not been in across subpopulations level. A contrast can shed light on the challenges that are familiar to both countries, it gives an emphasis to exclusive challenges that each country are encountering. We also found a gap where we decided to check the impact of patient cooperation, provider competency and resources and facilities on the health care system quality.

This study explains the basic core of health care sectors, economy and body of knowledge. As far as health care systems of China and Yemen are concerned, this research guides their management to devise wellbeing plans for their patients by developing more patient safety and quality services. Furthermore, service providers can also be facilitated through this research as they can have a glimpse that how their services can help them out in improving their individual performance. The consequences of this study motivate all health care systems segments of economy to equip them with state of the quality services and facilities in order to excel in competition with the health care standers. Finally, this study is beneficial for given knowledge to enhance the empirical evidence on many uncover areas especially because no study has been conducted regarding the comparison of health care systems in this two countries China and Yemen. have also suggested to analyze such comparison in developing countries like China and Yemen in health care sector. For the purpose of comparing china and Yemen health care system, we analyze the difference of health care quality system of China and Yemen, which correlate closely to the impact of patient safety on the health care systems, impact of provider competency on health care systems. A comparative efficiency in Medicare systems of China and Yemen was made to assign recommendation about the improvement on both countries health care systems.

\section{Literature review}

\section{a) The brief history of Chinese Health care system}

Later on the foundation of China in 1949, the country getting better after a long time suffering from both externally and internally disagreements confusion. As a consequence, the Chinese healthcare circumstances had decreased to a threshold level in contrast with other nations at a comparable rank of growth (Organization, 2012). During this era, the decision-making socialist Party supported the typical 20th century socialist ideology as well as assumed that the citizens, characterized by the government, must mutually possess all methods of making; there was no function for the private sector. Consequently government possessed the finance and start to operated facilities, involving big hospitals in the main cities and undersized rural community clinics at a countryside, suppliers were employees of the nation. In the rural areas, the community was the foundation of all features of life. Communities, is a significant organizations which symbolized the peasants, possessed the ground and controlling activities, involving agricultural, allocating products, providing social services for example health care and education (Al Juffali et al., 2018). Health care was given in the Cooperative Medical System (CMS), which was typically funded by means of a commune's combined returns and was plainly helped by the central government in the shape of inexpensively medicine and tools. CMS administrated village as well as rural community health clinics that were employed generally by practitioners who had only essential health care guidance. Chinese was utilizing a barefoot doctors which established many public relations and admire in the West for their believed effectiveness in congregating the requirements of rural inhabitants (Blumenthal, 2015). Chinas intensive care unit beds and the ICU high training nurses still inadequate, in 1980s the critical care medicine began in china with a single bed was opened in 1982 at Peking unio medical college hospital, until the end of 1989 when new regulation considered the Icu as a mandatory for the hospitals(Qiuet al.2001; wang and ma 2006). Even though the economy developed very gradually, Chinese health system obtained huge developments in healthcare sector, statistics in 2011 show that china has estimated by 30000 healthcare facilities. In (1950 -1990), Life anticipation approximately rising much high from, moreover there is a remarkable fall in newborn mortality (declining from 200 to 34 per 1,000 live births) (Hsiao, 2013). Such a developments does agreed with most important investments 
in public health in the course of a greatly centralized governmental society structured on the Soviet Union's system of near the beginning 1950s (Liu, 2012). In specifically, health care delivery was prepared as a three-tier, bottomup deliverance system. At the bottom level, rural village or urban street health centers gave fundamental preventive and remedial care and submitted patients who required supplementary action to rural community or community health centers. District or regional hospitals gave particular care to the sickest patients in the course of a widespread a hospitals network in both town and countryside (China, 2011). In addition, unusual concentration was paid to training health care employees. Thousands of "village doctors" (i.e., the previous barefoot doctors), were preferred by region health controls to obtain three to four months of early training in addition to added, yearly training to improve their talents (Hsiao, 2013).

\section{b) Difficulties and deficiencies faced by Health care system of China}

The citizens of China have being investing higher on the health care facilities. Thus, critical care medicine is the poorest disciplines of clinical medicine in china, needs more fund to develop the ICU including the intensive care nurses training. The health expenses rose from eleven billion Yuan (three per cent of the gross domestic product) in 1978 to eight hundred billion Yuan (six per cent of the gross domestic product) in 2018 (Tan, Liu, \& Shao, 2017). Consequently, the quality as well as extent of the health care systems enhanced a lot in the last 20 years. On the other hand, inequality in the wellbeing and health care services has become a vast and major issue since the restructuring of the health care facilities in China. Ineffectiveness has also become a problem that is not able to be ignored. On the whole, the restructuring of the health care system in China is ineffective (Tan et al., 2017; Wang et al., 2018).

\section{C) The brief history of Yemen Health care system}

In Yemen, traditional medicine was playing a major role in public health care, which was only the medical assistance available for people who seek treatment. that until the Yemen revolution in 1962, modern medicine was simply implemented. In 1990 after the Yemen unification, the government started to be concerned about public healthcare. the system was structured based on the tow programs the first is health centers and unit at the first touchline, the second is the prevention against epidemic and non epidemic disease. generally speaking no established experiences between these two parts. Yemen's health system is at the bottom of worldwide healthcare "Poverty, frequent pregnancies with no complaints about the closely period, and the least knowledge of healthcare" unite to set up off the little low newborn weight is the highest in the region (UNICEF, 2011). In 2014 the Yemen healthcare system shortfall was appeared during the spreading quickly of cholera epidemic due to a lack of clean water or health access, a thousands of people have died. Shortages in medicines and supplies is "persistent and widespread," WHO said, adding that health workers have not been paid in nearly a years. The expansion cause a multiple disabilities in Yemen's healthcare, one of the reasons is the way of life modifications, bad lifestyle, drinking unclean water, a build-up of human waste which is not being collected in major cities, and deficiency of sufficient care by the hospitals careless of the outpatient safety elderly were certainly probable than youth cohort to undergo a continual diseases such as heart disease, diabetes etc. Yemeni population also occurrence of material mistreatment mostly in the shape of tobacco usage In accordance with a study by (C. Scheifele et al (2013), "A) notable widespread presence of oral pre-cancerous lesions, such as oral squalors cell carcinoma with $27 \%$ in Yemeni people who's using Khat and Shamma tobacco. In sight of rising trouble of health care associated facilities (MoPHP) comprehended that the public sector on its own is no longer competent of giving essential health care for the people, that reason led to a large degree of privatized the sector. Patient security is a significant constituent of health care quality, It involves the measurement of patient safety features which is peak precedence in industrial countries nowadays; on the other hand, the patient safety features are not finely valued in the rising economies such as Yemen. As a consequence, lately there has been an enormous tour by Yemeni nationals in the appearance of medical tourism in other rising countries like India.

\section{d) Difficulties and deficiencies faced by Health care system of Yemen}

Health care services attain and even delivery of the medical care facilities have long been issues in the third world developing country like Yemen, although the Yemeni establishment certifies the right to the health care services for all the citizens of Yemen. Approximately thirty-five percent of people in Yemen do not get availability in the time of necessity and reach the healing facilities, it is restricted to nearly sixty-eight percent of the populace, while thirty-two percent are having a dearth of medical facilities wholly (Slama et al., 2016). But after war in 2011 there is a huge number estimated $50 \%$ of population are getting difficulty to access to the Hospitals(WHO). The allotment of such health care resources in Yemen is inclined to unfair outcomes as thirty percent of the whole health expenses move to the medical treatment out of the country for a slight figure of patients and the people of Yemen who have enough money to acquire the travel expenditures. Moreover, there is a geographic inequality in the allocation of availability to the health care facilities including the roads. Around twenty-five percent of the rural regions have availability, as contrasting to the eighty percent of urban regions. Furthermore, as the majority 
of the GHS (Government Healthcare System) hospices, as well as private medical clinics, are extended in the urban regions, few seventy percent of the entire populace does not have a convenient reach to the expert medical care and would experience a huge expenditure in the process of traveling to the city medical care amenities (Mihai, Jordanova, Volpe, \& Sartorius, 2016; Slama et al., 2016). Due to the weak economy of Yemen, health financing has put a great effort to finance its medical care services. In the year 2017, the whole health care expense in Yemen was 1.2 billion US dollars (sixty USD per capita), while the government of Yemen simply donated 4.6 million US dollars out of this which becomes nearly twenty percent per capita of Yemen. Furthermore, patients who avail the public health care facilities have to reimburse the consumer bill at the time of accessing the medical service and buy the given tablets out of pocket, both of which lead to a huge load when patients require the long-standing health care for serious diseases (Mihai et al., 2016). Compounding this issue, informal costs are frequently alleged by the health care service providers, who consider this act necessary as they are poorly paid and constantly underequipped (Organization, 2017b).

\section{e) Patient safety and health care system quality in china and Yemen}

The objective of this research is to examine the relationship between patient safety and health care service quality in China as well as in Yemen. A study was conducted from patients' insights and their association with the patient safety context and health care provider in hospitals. According to WHO's 2000 says; one of the major goals of health systems is to respond to patients expectations: In particular, people have a right to assume that the health system will treat them with individual dignity . their needs should be immediately attended to, without long delays in waiting for diagnosis and treatment - not only for better health outcomes but also to respect the value of people's time and to reduce their anxiety. Patients also often expect confidentiality, and to be involved in choices about their own health, including where and from whom they receive care. According to our survey data collection in Yemeni, there was widespread dissatisfaction, mainly because of the above reasons, and the poor staff attitudes and interpersonal skills included corruption among hospital staff, lack of utilities such as water supply, was driving patients to seek better services going to capital city hospitals. Furthermore, Yemeni people lost their trust relationship with health care services. Meanwhile, Chinas healthcare has shifted from preventive to curative care many public health services, and patients right is already fundamental for health care. Earlier researches performed in China have demonstrated direct linkage amid the health care service quality and patient safety as well as satisfaction. The patient safety, as well as reliability, is both an approach and trustworthy conduct. Thus, the patient response was much better than the result gotten from Yemen. Safety culture in the healthcare system still needs to develop in both countries "If the healthcare personnel do their job well, but the patient does not follow medical orders, the objectives would not be achieved" (CC, 2015). Following, Vogus et al. (2010), "In Yemen, it is explicitly distinguished between safety culture and safety climate by defining safety climate as the shared values and beliefs regarding safety efforts whereas safety culture encompass the climate as well as the actual behavioral norms and practices that delivery that safety and enhance the quality of health care system. It is considered to employ a safe environment as the base for rising patient safety and the health care system. It is within a given climate that the cautious function of a quality system may productively take place within the health care sector.

\section{f) Provider competency and health care system quality in China and Yemen}

There is inequity in both provider's competence and quality services. The concept of inseparability characteristic and interpersonal feature of service quality in China, within the research authors would believe the shared significance of patients' and service providers' views. Developing such shared strategy toward service quality will create requirement to cope with a gap that is inconsistency between patients' and service providers' insight of service quality as well as healthcare system quality (Miranda, 2014). According to the author by taking Yemen into consideration, "It should be considered that healthcare services as well as general services are existing for customers' satisfaction and even if healthcare service providers have their own essential opinion on healthcare service quality, they should always keep in mind that the core place is allocated to customers and direct their strengthens to deliver their services in line with their expectations and needs as well as it is in general service industry" (Scotti, 2012). The quality of existing services in Yemen is poor, particularly in the public sector including the health workers, and this contributes to the country's poor health outcomes. The reasons of why patients and health workers' response was unsatisfactory with the current quality in Yemen are plagued by overall supply, poor quality includes the lack of inputs for providing services low morale as a result of salary and wage pressures. unavailability of drugs and medical supplies, the lack of regulation, poor maintenance of facilities and equipment, standards distribution of staff not based on need. China Healthcare system output is a healthcare service provider's quality, so it should fit in traits of the general service quality. in china also some response shows unhappy react on the overuse of high-cost technologies and expensive medications. Liu and Mills (2005) examined detailed records of appendicitis and pneumonia patients and found that more than one-third of drug expenditures were unnecessary. Over prescription and unnecessary interventions are observed in Yemen as well especially antibacterial drugs. Moreover, care provided by the public sector is repeatedly described as poorer compared with 
china(Bhatia and Cleland, 2004; Mills et al., Intermediate Outcomes: Access, Quality, and Efficiency 212002 ). In summary, evidence shows that supply-induced overuse of medicines and procedures is a serious problem and that systematic health care quality assessments and controls need to be incorporated into the health care systems of both countries, Routine evaluation and assessment of quality is an important part of a health system in both countries. Nonetheless, this reality could be quite important in cases of making quality management schemes, for the purpose to become capable to obtain deeper facts concerning service quality within the healthcare system and be capable to cope with the illustrated gap, it is rational to take a glance at the healthcare service itself.

\section{Methodology}

This study will be an inductive study that will be conducted both quantitatively and qualitatively by means of interpretive approach. It will be an explanatory type of research. It utilizes primary data gathered by questionnaire and structured interviews to obtain findings related to hypothesis. The primary data was gathered by performing interviews and filling questionnaires from the health care sectors of China and Yemen to check their services, quality and patient safety and to see which respective sector is efficient in its operations. The sample of respondents was selected by means of purposive sampling and after that questionnaires and structured interviews was responded by these respondents. For quantitative survey, 5 surveys with the help of questionnaire and the interviews was conducted. then the data will be analyzed for interviews through framework analysis and for questionnaires, it was analyzed by using SPSS and by performing tests of reliability, confirmatory factor analysis to check the validity and model fitness of data. For questionnaire formation, scale used for patient safety was adopted of the author (Gardner, 2012), while for provider competency, scale was adopted of the author (Anna Globenko, 2012). The data was collected through both primary and secondary ways to improve the validity of this research paper. Investigators have enlightened that questionnaire contains a number of different questions, both open-ended and close-ended, related to the research topic. Different options are also given along with the questions and respondents chose the best option which suits them. In this study, a self-constructed questionnaire was used to obtain the data from the sample characterize the option into $0,1,2,3,4$ and 5 representing poor/ bad/ good/ satisfied/ very satisfied. For qualitative surveys, the respondents were divided into two groups. The respondents of group A of 150 size were highly educated know the standard of medical facilities. We also provided the basic training to group A respondent regarding to the parameters and standards, on which they conducted the evaluation of surveys. The group B (sample population) also conducted the same evaluation Each questionnaire contained 12 questions in total, and 9 questions were in objective phase and 3 questions were in demographic phase. The total 150 questionnaires form controlled volunteers (A group) and sample population (B group) were filled out from local people of china and Yemen though different mediums and then were overseen by the researchers to check its validity for each parameter. The ethical values were observed in this study proficiently. At first, the response rate from both group and the privacy of respondents was also maintained and the data collected from them were not made public.

\section{Results and Discussion}

Although china is our model to enhance and improve Yemen health care system, china health outcomes still been categorized as one of the countries which need more scaled up their healthcare level while comparing with other developed countries. By the estimated a millions of people does not have a health insurance because the prohibitively of high insurance expenses hindering them from getting proper healthcare, most of them are from rural and countryside. Speaking of the world infant mortality china is the $20^{\text {th }}$, low-birth weight is the number $29^{\text {th }}$, and the life expectancy is the $6^{\text {th, }}$ which making it the highest in an industrialized countries. According to quantitative data analysis, 550 patients were admitted during 3 month in china, versus 120 patients were admitted in Yemen. Among these patients, $49 \%$ were above 60 years old, most of the patients were treated with Medicare (47\%) while $20 \%$ were treated with medic aid in China. In Yemen, 36\% of total patients was above 60 age, which is significantly lower than china (Table 1). It reflects that disease dispersal were quite common in young ages in Yemen, It might be due to effects of civil war, financial and political disturbance in Yemen which affected on health of even young people. The ratio of medic aid provision was 5\% percent less in Yemen and average stay of 10 days as compared to 5.5 days in China. We also made comparison for maintenance of digital record by selective interviews from the concerned person. The record maintenance system in Chinese hospitals is found to be international standard, and almost complete computerized bio data regarding to medical provider information, clinical notes, detail problem list of patients, discharge and transfer summaries were almost found to be very high (Figure1). The qualitative analysis (Figure1) illustrates the Yemenis effort on healthcare reforms, it is synchronizes with Chinese reforming their own healthcare system. As compared to China, the digital records of these parameters were found to be below $40 \%$ which need to be improved substantially in order to keep the proper record of patients. The digital record of individual service plans of all admitted patients were found to be little less $(80 \%)$ and found to be significantly better than Yemen (40\%). It's worth to noted here that In Yemen, We found the health assisted projects of ICRC and WHO delivering supplying fuel to keep electricity generators functioning for operating 
theatres and for the country's blood banks labs, and petrol for ambulances( Shadoul) says "WHO has provided the fully supports with new attitude toward all the up and running activities including the financial cost, and an ambulances vehicle establishment subsidized with global positioning systems technologies.

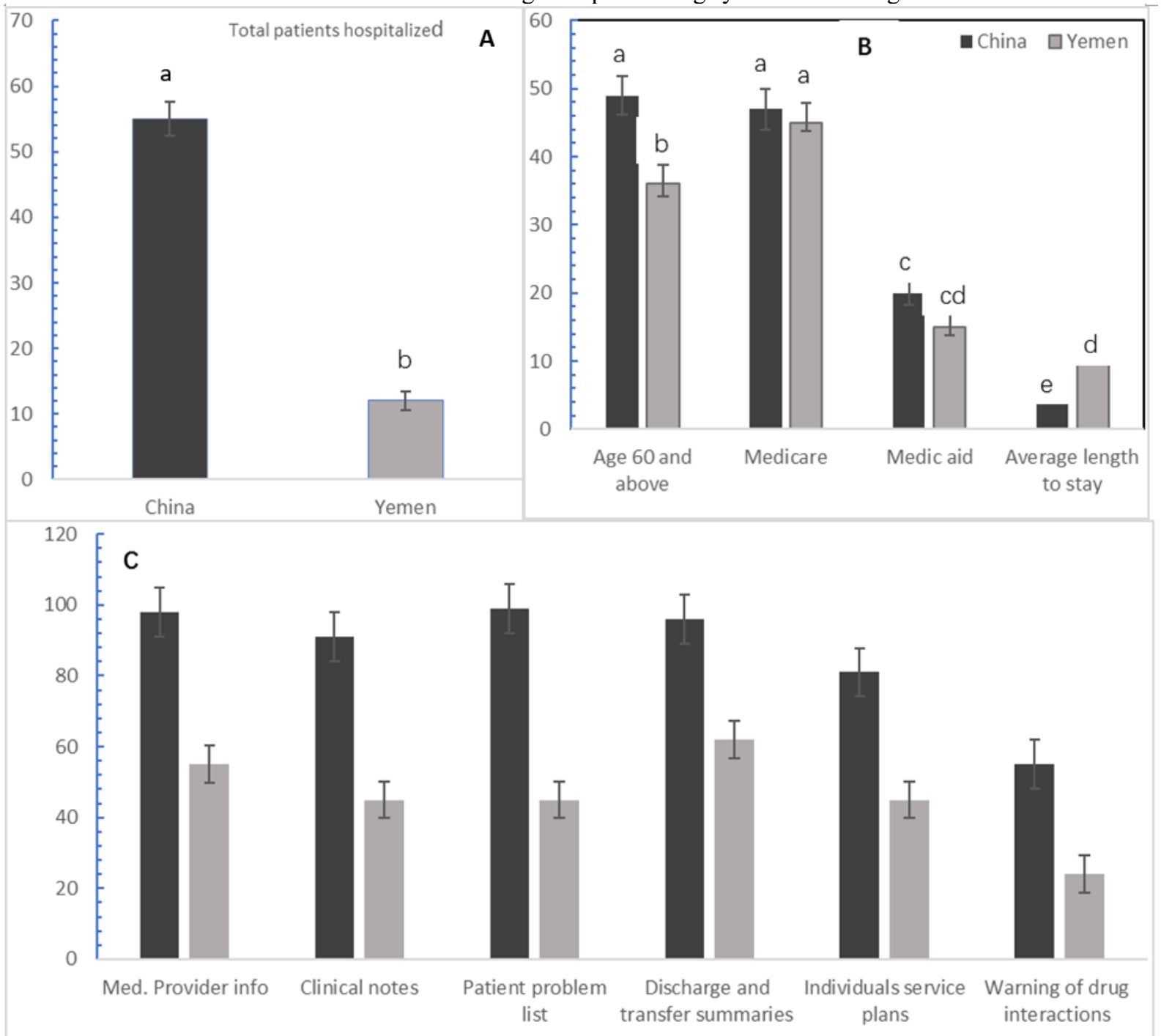

Figure 1. It represents quantitative data obtained from direct interviews from the staff in selected hospitals of China and Yemen. A. it shows the total patients admitted during the year. B. The information about the patients treated in the hospitals. C. its express the percentage of digital maintenance record of patients. The test of significance was calculated by at $0.05 \mathrm{P}$ level and bars shows the errors according to standards error deviation.

\begin{tabular}{|c|c|c|c|c|c|c|c|}
\hline \multicolumn{8}{|c|}{ Control volunteers } \\
\hline & \multicolumn{2}{|c|}{ gender } & \multicolumn{3}{|c|}{ Age } & \multirow{2}{*}{ Total } & \multirow{2}{*}{ response rate $\%$} \\
\hline & male & females & $25-40$ & $40-59$ & above 60 & & \\
\hline China & 78 & 72 & 45 & 55 & 50 & 150 & 100 \\
\hline Yemen & 123 & 27 & 55 & 65 & 30 & 150 & 100 \\
\hline \multicolumn{8}{|c|}{ Sample group } \\
\hline & \multicolumn{2}{|c|}{ gender } & \multicolumn{3}{|c|}{ Age } & \multirow[t]{2}{*}{ Total } & \multirow{2}{*}{ response rate $\%$} \\
\hline & male & females & $25-40$ & $40-59$ & above 60 & & \\
\hline China & 71 & 79 & 23 & 60 & 67 & 150 & 96.66 \\
\hline Yemen & 45 & 105 & 55 & 65 & 30 & 150 & 93.33 \\
\hline
\end{tabular}

Table 1. It represents the total respondents od control and sample group for qualitative data analysis

Questionnaire survey conducted from a controlled and sample group revealed the sound comparison between china and Yemen. Doctors experienced, qualified behavior are the first outcomes of patients satisfaction. Moreover, Poor health is associated with worse physical and mental well-being. The Disruptive behavior of doctors and nurses are dangerous leads to increase complications environment, medication errors, and make a difficult to 
determine the extent of problem. The provider competence is important factor in healthcare, survey showed its one of the reason behind the healthcare quality and getting patients satisfactory it is incrementally long term relationships among health workers and patients is the only way to obtain the mutual trust. Low salary for healthcare workers is the main reason for not getting motivation to provide good care for patients, this purpose could be achieved by promoting best practices, increasing the financial fund on the healthcare system, more for developing professional norms of behavior. Patients in Yemen expressed their dissatisfaction toward current healthcare providers, there is $55 \%$ from a sample population, and $45 \%$ from controlled group expressed doctors and paramedical behaviors were very bad and disruptive. While regression correlation found no difference between controlled and sample population (Figure 2). Poor staff attitude is one of the main barriers for accessibility of healthcare(Ovetveit J, AL-serouri A2005). Meanwhile, in china Patients considered doctors and paramedical behavior as quite friendly. China enhanced medical safety and better protect patients health right ahead of the first world patient day, china's healthcare system contain the ability to coordinate, cooperate and communicate with regard to both patient safety and provider competency.

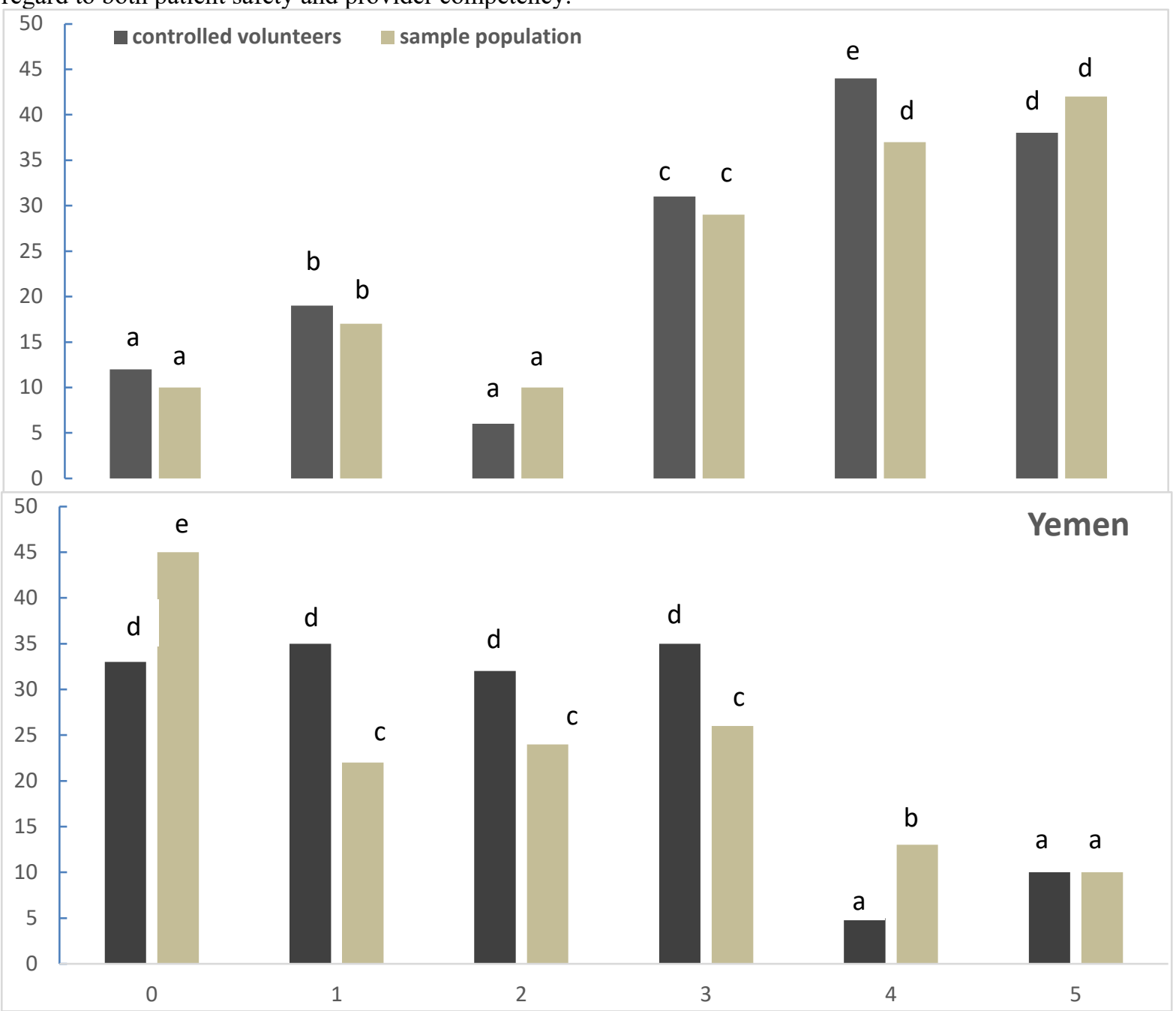

Figure 2. It expresses the staff (doctors, paramedical staff and nurses) behavior and expertise examine to patients. The correlation was checked with logarithmic regression at $0.05 \mathrm{P}$ level.

Least developing countries like Yemen, usually the healthcare system still remained well short of needs. public hospital cannot enable to address the people urgent health needs, Unfortunately, there is a greater need beyond affordable care capacity. It's essential to remark the numbers of Yemen health care workers such as doctors, medical assistants and nurses which had shown a severe shortage [Figer3]. There is a total of 3,788 physicians and 9,419 nurses in Yemen which represents a national ratio of 0.23 physician and 0.55 nurse per 1,000 population(East, M., \& Region, N. A. 2000Comprehensive Yemen). This underscores the lack of an effective human resource management strategy and the difficulty of effective management under the civil service constraints. Moreover, there are wide regional variations in manpower distribution as exemplified by the presence of almost $50 \%$ of all physicians in Aden \& Sana'a. Additionally, there is lack of adequate training and continuous medical education (East, M., \& Region, N. A. 2000). In Yemen, all possible efforts aimed at attaining one goal, which is increasing 
healthcare availability developing the access to better health services by offering competence providers. While in China, the crucial importance was preserving citizens from a financial shocks which stem from medical expenses in the countryside rural area. According to statistic showed a number of licensed doctors in china amounted to 3.6 million in the year 2018(Lai lin Thomala), beside that china's has more than 88000 rural doctors, about 20000 assistance doctors and health workers(Dr. zhang zho yang). that's numbers is putting china above the East Asia and Pacific average. Despite the efforts were Chinese government made but much remained to be done, healthcare is costly. For instance, financial shocks might be a real catastrophe for people when facing a medical condition or any indisposed. Chinese government started to motivated their citizens who could to get health insurance trying to increase the numbers as many people as it possible by a concrete moves, such as direct subsidies for all public officials, agents and employees of the state.

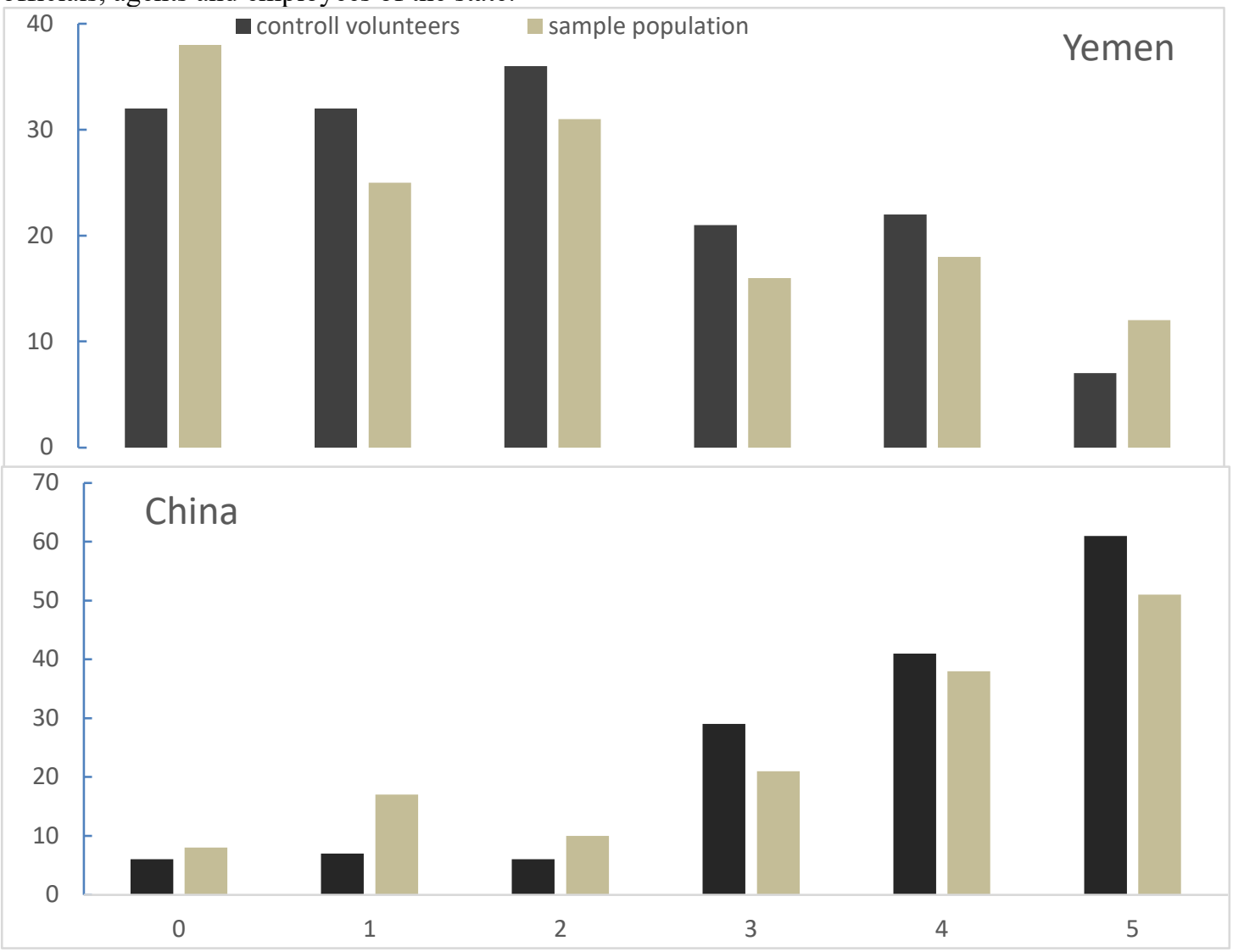

Figure-3. It expresses the staff (doctors, paramedical staff and nurses) availability. The correlation was checked with logarithmic regression at $0.05 \mathrm{P}$ level.

In Yemen based on the recent Health Resources and Services Availability Monitoring System (HeRAMS) estimation of 2242 health facilities, the public and the privet general hospital were around 200 with an insufficient equipment. The shortage of approachability to the equipped health facilities, and the government deficiency in finance were existed even before the war started. In the countryside there only $24 \%$ of residents could access to health facilities, and in all areas was about $42 \%$. This unavailability due to limited geographies coverage, and also by the poor payment system which required to receive in cash. After 2011 the war engulfed the entire country and only 10 per cent of health facilities surveyed across 14 governorates had sustained partial or full damage it's almost impossible to practice good medicine and to provide modern standards of care. China is a manufacturing state, so health facilities considered to be of superior quality and the most technologically advanced compared to Yemen. It had more than 32000 hospitals and the majority of hospitals are public. A thousands of tertiary hospitals, secondary hospitals and primary hospitals with a total of 8.4 million sickbeds its 5.8 percent up year-on-year. Chinese domestic medical device companies are consolidating upgrading quality and beginning to compete in medium level technology niches. 


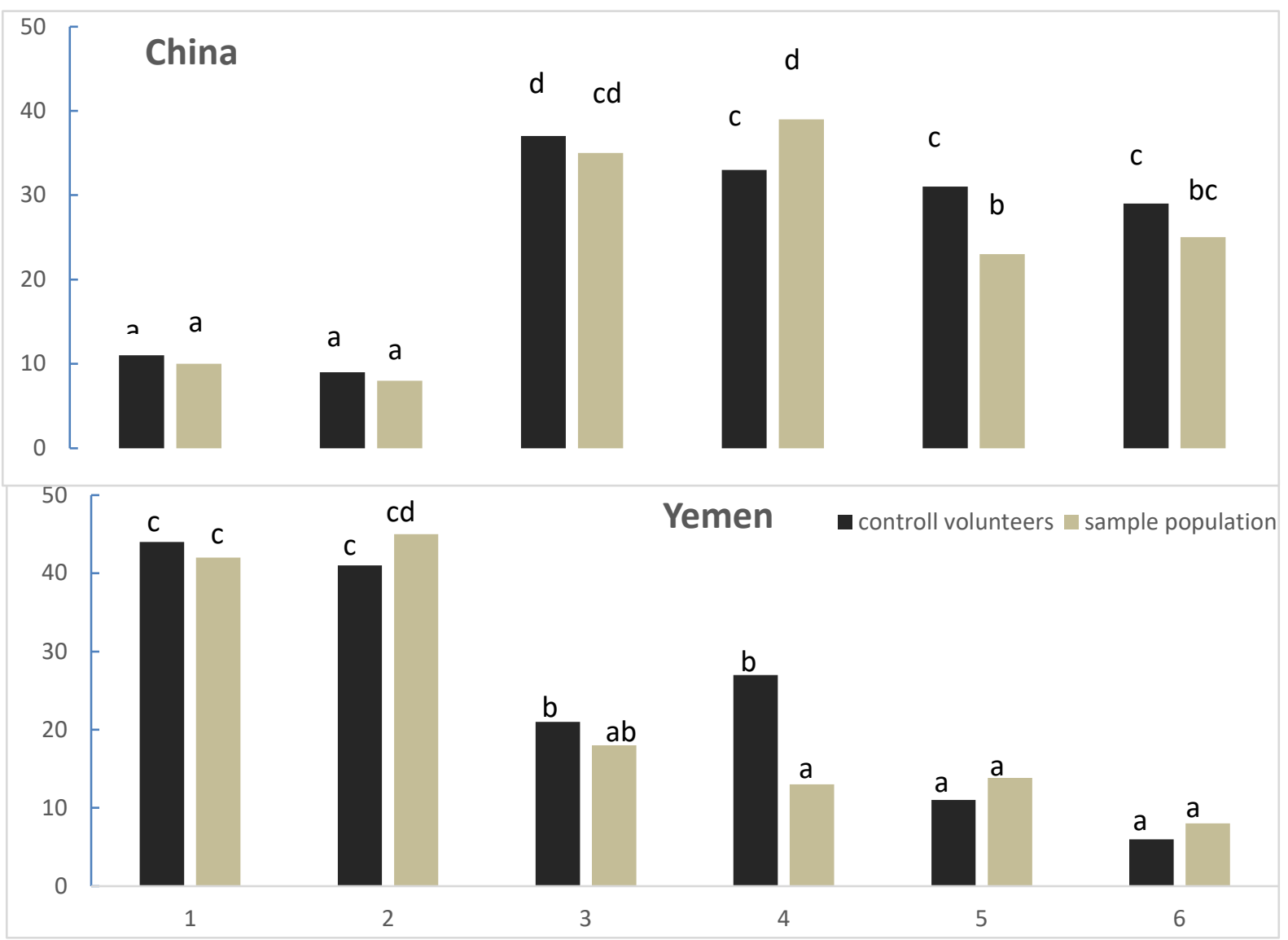

Figure-4. It expresses the availability of equipment in hospitals. The correlation was checked with logarithmic regression at $0.05 \mathrm{P}$ level

\section{Conclusion}

The overarching lesson that can be learned from comparing the healthcare systems of China and Yemen is that a differences in incomes have been driven the countries to made a varying quality of medical care providers, and the local conditions also play pivotal role in creating a nation's healthcare reforms. Thus in return, the stable efficiency of healthcare system is a significant factor in establishing a stable economy. China has been focused on improving people's health standards, providing an easy access to hospitals with proper Medi-care and medic aid facilities in rural area, and patients expressed their satisfaction versus Yemeni patients expressed their dis-satisfaction of their over healthcare quality. Chinas hospitals are adapted the computerized digital record system to maintain the proper record clinical notes, patient problem list, individual service plans and detailed information about medication provided in hospitals, which is help to provide a quality health service on patients safety, while Yemen is far below from the trend line of digital recoded maintenance. In regard to patient safety and according to the survey Yemeni people want a big amendment changes in the country healthcare system to get tangible results. Yemen health care system is poorer and people access to hospitals significantly less than China, the availability of medical staff, doctors and medical equipment's are found to be a serious issue in Yemen hospitals while in China people moderately considered it satisfactory. The study of Chinas health care system is an ideal model to offers a particular lessons for Yemeni health policymakers .

\section{Acknowledgments}

The author is grateful to the professor Zong Mao Fu school of health science Wuhan university, who assisted with helpful and detailed information

\section{References}

Al-Murtadha, M., \& Feryok, A. (2017). Studying English in Yemen: situated unwillingness to communicate in sociohistorical time. Innovation in Language Learning and Teaching, 11(3), 230-240.

Al Juffali, L., Al-Aqeel, S., Knapp, P., Mearns, K., Family, H., \& Watson, M. (2018). Using the Human Factors Framework to understand the origins of medication safety problems in community pharmacy in Saudi Arabia: A qualitative study. Research in Social and Administrative Pharmacy. al, S. M. e. (2013). 
A comparison of the health systems in China and India. RAND-centre for Asia pacific policy.

Alfonso, C. A., Summers, R. F., Kronfol, Z., Jiménez, X., Winanda, R. A., \& Tasman, A. (2019). Psychiatry Residency Education in Countries with Low-and Middle-Income Economies Advances in Psychiatry (pp. 697-722): Springer.

Alssamei, F. A., Al-Sonboli, N. A., Alkumaim, F. A., Alsayaad, N. S., Al-Ahdal, M. S., Higazi, T. B., \& Elagib, A. A. (2017). Assessment of Immunization to Hepatitis B Vaccine among Children under Five Years in Rural Areas of Taiz, Yemen. Hepatitis research and treatment, 2017.

Anna Globenko, Z. S. (2012). Service quality in healthcare: quality improvement initiatives through the prism of patients' and providers' perspectives

Baidoun, S. D., Salem, M. Z., \& Omran, O. A. (2018). Assessment of TQM implementation level in Palestinian healthcare organizations: The case of Gaza Strip hospitals. The TQM Journal, 30(2), 98-115.

Balan, P., He, H.-G., Cao, F., Wong, M., Chong, Y.-S., Lopez, V., . . . Seneviratne, C. (2018). Oral Health in Pregnant Chinese Women in Singapore: A Call to Go beyond the Traditional Clinical Care. Paper presented at the Healthcare.

Blumenthal, D., and W. Hsiao. (2015). Privatization and Its Discontents-The Evolving Chinese Health Care System,. New England Journal of Medicine.

BUNEVICIUS, R., KUSMINSKAS, L., BUNEVICIUS, A., NADISAUSKIENE, R. J., JURENIENE, K., \& POP, V. J. M. (2009). Psychosocial risk factors for depression during pregnancy. Acta Obstetricia et Gynecologica Scandinavica, 88(5), 599-605. doi: doi:10.1080/00016340902846049

CC, Y. (2015). The impact of human resource management practices on the implementation of total quality management: An empirical study on high-tech firms. The TQM Journal.

China, P. T. o. t. D. R. C. o. t. S. C. o. (2011). "An Evaluation of and Recommendations on the Reforms of the Health System in China. China Development Review.

Cunningham, A., Ingram, W., Brinckmann, J., \& Nesbitt, M. (2018). Twists, turns and trade: A new look at the Indian Screw tree (Helicteres isora). Journal of ethnopharmacology, 225, 128-135.

Gardner, J. W. (2012). Improving Hospital Quality and Patient Safety An Examination of Organizational Culture and Information Systems.

Hsiao, W. C., and P. S. Heller. (2013). What Macroeconomists Should Know About Health Care Policy. Washington, D.C.: International Monetary Find.

Lee, M. C., Hall, B. J., Bulterys, M., Ling, L., \& Tucker, J. D. (2014). Improving health services for African migrants in China: A health diplomacy perspective AU - McLaughlin, Megan M. Global Public Health, 9(5), 579-589. doi: 10.1080/17441692.2014.908935

Liu, Y., W. C. Hsiao, and K. Eggleston. (2012). Equity in Health and Health Care: The Chinese Experience. Social Science \& Medicine,

McCann, T. V., Mugavin, J., Renzaho, A., \& Lubman, D. I. (2016). Sub-Saharan African migrant youths' helpseeking barriers and facilitators for mental health and substance use problems: a qualitative study. BMC Psychiatry, 16(1), 275. doi: 10.1186/s12888-016-0984-5

Miranda, F. J., Chamorro, A., Murillo, L.R. \& Vega, J. (2014). Assessing primary healthcare services quality in Spain: managers vs. patients perceptions. The Service Industries Journal.

Monge-Maillo, B., López-Vélez, R., Norman, F. F., Ferrere-González, F., Martínez-Pérez, Á., \& Pérez-Molina, J. A. (2015). Screening of Imported Infectious Diseases Among Asymptomatic Sub-Saharan African and Latin American Immigrants: A Public Health Challenge. The American Journal of Tropical Medicine and Hygiene, 92(4), 848-856. doi: https://doi.org/10.4269/ajtmh.14-0520

Mozooni, M., Preen, D. B., \& Pennell, C. E. (2018). Stillbirth in Western Australia, 2005-2013: the influence of maternal migration and ethnic origin. The Medical Journal of Australia, 209(9), 1.

Nee, A. Y. H. (2018). Positioning Malaysia in Medical Tourism: Implication on Economic Growth in ASEAN Integration. Indonesian Journal Of Business And Economics, 1(1).

Organization, W. H. (2012). The World Health Report 2000-Health Systems: Improving Performance.

Scotti, D. J., Driscoll, A.E., Harmon, J. \& Behson, S.J. (2012). Links Among High- Performance Work Environment, Service Quality, and Customer Satisfaction: An Extension to the Healthcare Sector. Journal of healthcare management.

Singer, S., Meterko, M., Baker, L., Gaba, D., Falwell, A., Rosen, A. (2013). Workforce perceptions of hospital safety culture: Development and validation of the patient safety climate in healthcare organizations survey. Health Services Research

Vogus, T. J., Sutcliffe, K.M, Weick, K.E.,. (2010). Doing no harm: Enabling, enacting, and elaborating a culture of safety in health care. Academy of Management Perspectives.

Whittaker, A., Chee, H. L., \& Por, H. H. (2017). Regional circuits of international medical travel: Prescriptions of trust, cultural affinity and history. Asia Pacific Viewpoint, 58(2), 136-147. 